\title{
Evaluación de la Inmunidad de un Motor de Inducción Monofásico frente a Huecos de Tensión
}

\author{
Luis F. Navarro, Joaquín E. Caicedo, Edwin Rivas y Francisco Santamaría \\ Universidad Distrital Francisco José de Caldas, Grupo de Compatibilidad e Interferencia Electromagnética - \\ GCEM, Crr 7 No 40B - 53, piso 5, Bogotá D.C. - Colombia (e-mail: Ifnavarroj@correo.udistrital.edu.co, \\ jecaicedon@correo.udistrital.edu.co, erivas@udistrital.edu.co y fsantamariap@udistrital.edu.co)
}

Recibido May. 2, 2013; Aceptado Jul. 19, 2013; Versión final recibida Sep. 18, 2013

\begin{abstract}
Resumen
Este artículo presenta una metodología para evaluar la inmunidad de un motor de inducción monofásico frente a huecos de tensión por medio de simulaciones en Matlab/Simulink. Para ello se diseñó un modelo de generador monofásico de huecos de tensión, y se implementaron algoritmos para obtener, modelar y mejorar la curva de sensibilidad del motor de inducción con respecto a las caídas de tensión. La metodología implementada es una guía sencilla para estimar el impacto de los huecos de tensión en motores de inducción reales. Adicionalmente, la metodología propuesta reduce la cantidad de huecos de tensión a los que se deben exponer dichos equipos durante las pruebas de laboratorio al usar otras metodologías.
\end{abstract}

Palabras clave: huecos de tensión, curva de sensibilidad, Matlab/Simulink, motor de inducción.

\section{Immunity Assessment of a Single-Phase Induction Motor to Voltage Sags}

\begin{abstract}
This paper shows a methodology for evaluating the immunity of a single-phase induction motor to voltage sags by simulations in Matlab/Simulink. For this, a model of a single-phase voltage sag generator was designed and algorithms were implemented, to obtain, model and improves the sensitivity curve of the induction motor with respect to the voltage drop. The implemented methodology is a simple guide to estimate the impact of voltage sags on induction motors. Additionally, the proposed method reduces the amount of voltage sags to which the motors are usually subjected in lab test using other methodologies.
\end{abstract}

Keywords:, voltage sag, sensitivity curve, Matlab/Simulink, voltage sag, induction motor 


\section{INTRODUCCIÓN}

Dentro de los disturbios más comunes asociados a la calidad de la potencia eléctrica (Afonso et al., 2007) se encuentran los huecos de tensión (sags), definidos en (IEEE Std 1159, 1995) como una disminución entre el 0.1 y el 0.9 por unidad (pu) del voltaje nominal, con duración entre 0.5 ciclos a 1 minuto, estos son causados principalmente por fallas en el sistema de potencia, cambios de carga y arranque de grandes motores (Caicedo et al., 2012; Su et al., 2013; Wang et al., 2011), y afectan el correcto funcionamiento de los equipos eléctricos asociados al usuario final del sistema de potencia (Bollen, 2000).

Key (1979), hace énfasis en la susceptibilidad de equipos electrónicos a las variaciones de tensión y el impacto económico que esto tiene en las industrias modernas. Wang et al. (2012) presentan un método para determinar el comportamiento del torque de un $\mathrm{Ml}$ al ser sometido a huecos de tensión. McGranaghan et al. (1993) realizan un análisis más detallado de las causas de los huecos de tensión y el impacto sobre equipos eléctricos-electrónicos fundamentales en la industria, como ASDs, PLCs y contactores de motor, lo que permitió establecer soluciones mediante la implementación de acondicionadores de potencia para mantener la tensión dentro de límites aceptables.

La evaluación de la inmunidad es un procedimiento que permite determinar la magnitud y duración de los huecos de tensión que ocasionan falla en un equipo eléctrico-electrónico (CIGRE/CIRED/UIE WG C4.110, 2010; Caicedo et al., 2011). Como resultado de la evaluación de la inmunidad se obtiene la curva de sensibilidad que describe el comportamiento del equipo frente a diferentes magnitudes de tensión con determinada duración.

Con base en los aportes realizados por la IEEE 493, Conrad y Bollen (1997) proponen técnicas para la coordinación de equipos sensibles, teniendo en cuenta mediciones y predicciones de las características de huecos de tensión. La principal contribución es el análisis teórico-práctico considerando los niveles de emisión e inmunidad de un entorno electromagnético determinado. Bollen (2000) propone una metodología para el análisis de compatibilidad electromagnética (CEM) para el caso de los huecos de tensión, revisando el tema desde tres focos principales: caracterización, desempeño de los equipos eléctricos y evaluación estocástica. Siendo esto último la principal contribución, pues se proponen dos métodos para la estimación de la ocurrencia de huecos de tensión: el método de posición de fallas y el método de distancias críticas.

En este artículo se presenta e implementa una propuesta metodológica para la evaluación de la inmunidad de un Motor de Inducción (MI) ante huecos de tensión. Para ello se desarrollaron esquemas en Matlab/Simulink, y algoritmos que permiten aplicar la metodología y obtener la curva de sensibilidad del MI frente a huecos de tensión. La metodología también propone un proceso para mejorar la precisión de la curva de sensibilidad empleando los índices de pérdida de tensión y pérdida de energía, lo que finalmente reduce tiempos de simulación y el número de huecos de tensión a los que se debe someter un equipo.

\section{MARCO CONCEPTUAL}

La norma IEC 61000-4-11 propone un método para determinar la tolerancia de equipos eléctricoselectrónicos a los huecos de tensión. Esta norma define un número deseado de magnitudes y duraciones de huecos de tensión a los cuales debe ser sometido el equipo bajo prueba (Bollen, 2000). Trujillo et al. (2007) proponen una metodología para evaluar el impacto de los huecos de tensión sobre equipos electrónicos de potencia.

La Fig. 1 muestra el esquema general para realizar la evaluación. El generador de huecos de tensión somete al equipo electrónico de potencia a huecos de tensión con magnitudes desde 108 Vrms hasta 12 Vrms, con descenso en pasos de $12 \mathrm{Vrms}$, y duraciones desde $5 \mathrm{~ms}$ hasta $1 \mathrm{~s}$, con incrementos de $5 \mathrm{~ms}$. El criterio para establecer la condición de falla se determinó con la tensión entregada por el equipo electrónico de potencia a las unidades conectadas: si la tensión de salida se alejaba $\pm 10 \%$ de la condición normal se decía que el equipo fallaba.

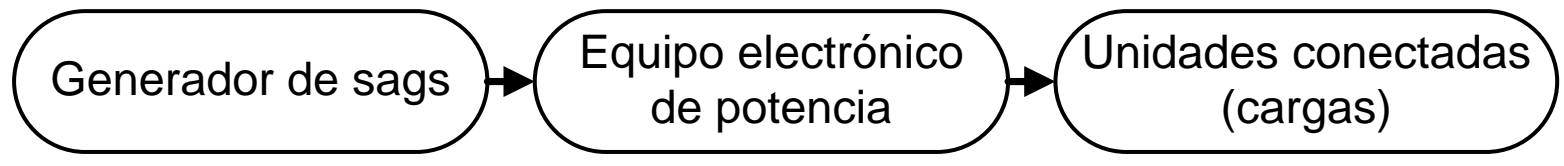

Fig. 1: Esquema general para evaluar la inmunidad de equipos electrónicos (Trujillo et al., 2007) 


\section{Índices para estimar el impacto de los huecos de tensión}

Algunos estudios estiman el impacto de los huecos de tensión sobre equipos eléctricos-electrónicos a partir de los índices de pérdida de tensión y pérdida de energía (Bollen et al., 2003; Leborgne et al., 2003). El índice de pérdida de tensión $\left(L_{v}\right)$ representa la diferencia entre el voltaje nominal del equipo y la tensión durante el hueco, integrado en el intervalo de tiempo de duración del evento, y se calcula con (1) (Leborgne et al., 2003).

$L_{V}=t_{t_{1}}^{t_{2}} 1-\frac{V_{\text {sag }}(t)}{V_{n}} d t$

Donde $V_{\text {sag }}(t)$ corresponde al voltaje durante el hueco, $V_{n}$ es el voltaje nominal del equipo, $t_{1}$ es el tiempo de inicio del hueco de tensión y $t_{2}$ es el tiempo de recuperación del hueco de tensión. El índice de pérdida de energía $\left(L_{E}\right)$ representa la energía no suministrada al equipo debido a la reducción de la tensión durante un hueco y se calcula con (2) (Bollen et al., 2003; Leborgne et al., 2003).

$L_{E}=t_{t_{1}}^{t_{2}} 1-{\frac{v_{\text {sag }}(t)}{V_{n}}}^{2} d t$

Cuando la tensión eficaz durante el hueco es constante, y su magnitud se expresa en por unidad (pu), los índices de pérdida de tensión y perdida de energía se calculan con (3) y (4) respectivamente.

$L_{V}=1-V_{p u} \times \Delta t$

$L_{E}=1-V_{p u}^{2} \times \Delta t$

Donde $V_{p u}$ es la magnitud del hueco de tensión en pu y $\Delta t=t_{2}-t_{1}$ corresponde a la duración del hueco de tensión. Para estimar el impacto de un hueco de tensión sobre un equipo eléctrico-electrónico con los índices de pérdida de tensión y pérdida de energía, es necesario un registro de los huecos de tensión que hayan provocado falla en el equipo, para ser tomados como valores de referencia.

La metodología expuesta por Trujillo permite obtener con mayor certeza la magnitud del hueco de tensión que genera falla sobre los equipos. El objetivo del presente trabajo es complementar esta metodología para optimizar la duración de las pruebas en equipos eléctricos-electrónicos, disminuir la cantidad de huecos de tensión a los que hay que someter los equipos bajo prueba y proponer un criterio para evaluar la inmunidad.

\section{METODOLOGÍA}

Para realizar una prueba de inmunidad bajo un ambiente controlado es indispensable un generador de huecos de tensión (Senturk y Hava, 2012), el equipo bajo prueba y un sistema de adquisición de datos (Tenaga Nasional Berhad, 2007). La Fig. 2 presenta el esquema general propuesto para una prueba de inmunidad.

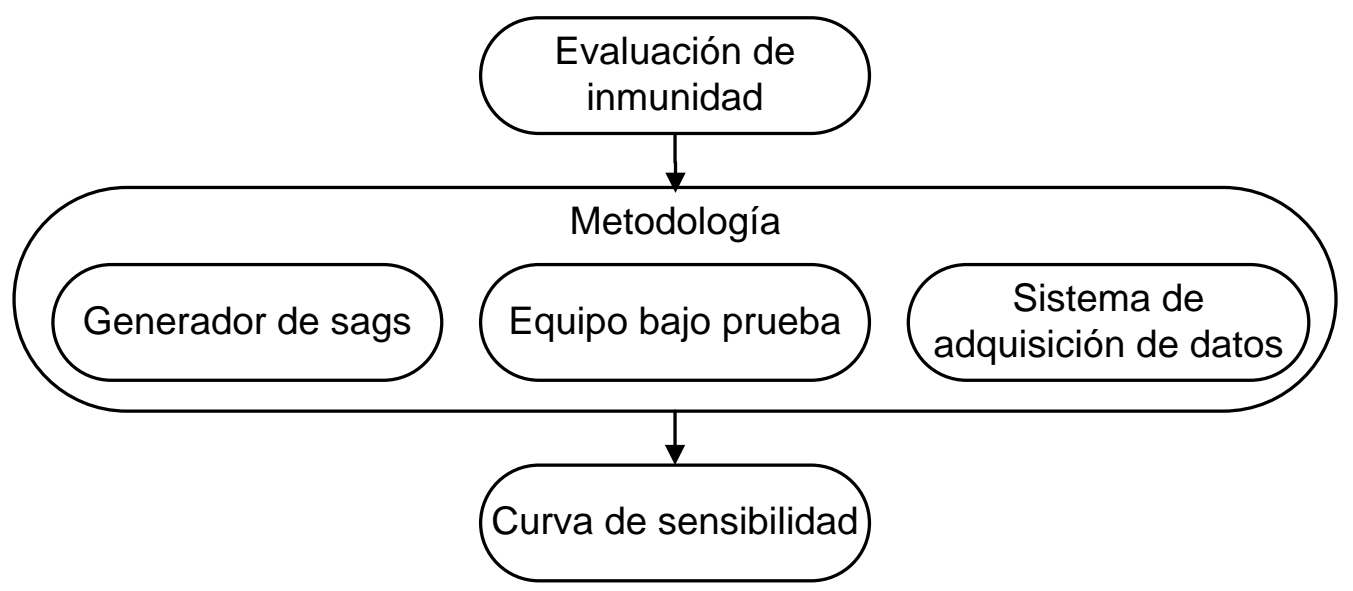

Fig. 2: Esquema general de una prueba de inmunidad 
La Fig. 3 muestra el esquema diseñado para evaluar la inmunidad del MI, el cual consta del generador de huecos de tensión, el MI como equipo bajo prueba, y el sistema de adquisición de datos que corresponde al conjunto de elementos utilizados para medir los parámetros de tensión, corriente entregados por el generador y la velocidad en el rotor del MI.

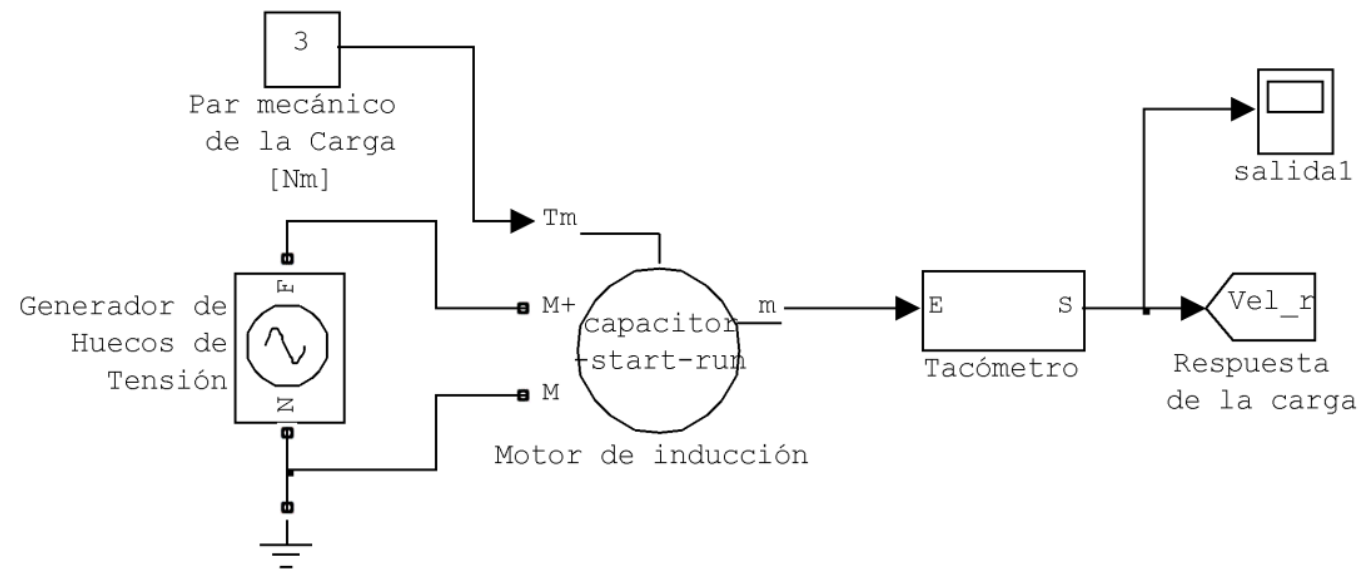

Fig. 3: Esquema de prueba

El modelo ideal del generador de huecos de tensión implementado (Fig. 4) provoca una disminución en la tensión mediante el cambio de una fuente de tensión con magnitud nominal $\left(\mathrm{V}_{\mathrm{n}}\right)$, a otra que representa la magnitud de la tensión durante el hueco $\left(\mathrm{V}_{\text {sag }}\right)$. La operación lógica ubica el selector de fuente en la tensión nominal para $t<t 1$ y $t>t 2$ (t1 y t2 son tiempo de inicio y tiempo de finalización del hueco de tensión respectivamente), y la tensión durante el hueco para $t 1<t<t 2$. El modelo permite observar los parámetros de tensión y corriente del generador, y la señal que indica el inicio del hueco de tensión.

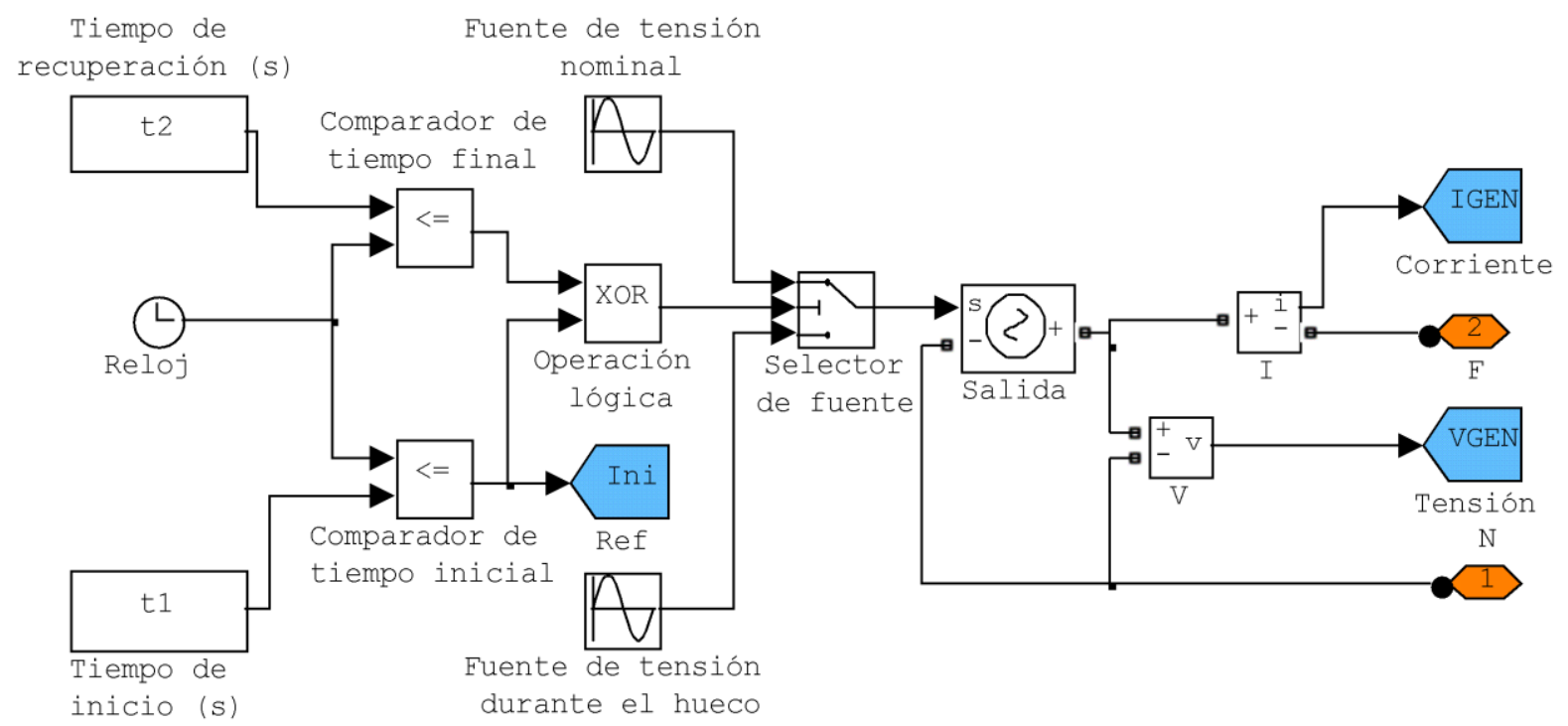

Fig. 4: Modelo ideal del generador de huecos de tensión

El motor de inducción es uno de los equipos más utilizados a nivel industrial, caracterizados por su sensibilidad las variaciones de tensión debido a que el par electromagnético es función del cuadrado de la tensión en el estator (Macri y Benedetti, 2012), el impacto que genera un hueco de tensión sobre un MI en operación, no solo puede detener un proceso, sino que también puede generar picos de corriente mayores a los del arranque por el toque que se presenta al momento de la re-aceleración ( Gómez et al., 2002), a su vez este pico de corriente puede afectar otros equipo conectados a la misma red.

Dado que la curva de sensibilidad de un equipo eléctrico-electrónico a los huecos de tensión, depende de las características del control y la aplicación del mismo (Trujillo et al., 2007), la curva de sensibilidad del MI la determina la carga mecánica y del rango de velocidad en el que debe operar para llevar a cabo un proceso. En la Tabla 1. Se resumen las características y condiciones de operación del motor de inducción monofásico utilizado para validar la metodología que se desarrolla en este artículo. 
Tabla 1: Características del motor de inducción monofásico

\begin{tabular}{|l|l|}
\hline \multicolumn{2}{|c|}{ Motor de inducción (equipo bajo prueba) } \\
\hline Potencia & $1 / 4 \mathrm{HP}-(186.5 \mathrm{~W})$ \\
\hline Voltaje & $120 \mathrm{Vrms}$ \\
\hline Frecuencia & $60 \mathrm{~Hz}$ \\
\hline \multirow{2}{*}{ Devanado del estator } & $\mathrm{R}_{\mathrm{S}}=2.02 \Omega$ \\
\cline { 2 - 2 } & $\mathrm{X}_{\mathrm{s}}=7.4 \mathrm{mH}$ \\
\hline \multirow{2}{*}{ Devanado del rotor } & $\mathrm{R}_{\mathrm{r}}=4.12 \Omega$ \\
\cline { 2 - 2 } & $\mathrm{X}_{\mathrm{r}}=5.6 \mathrm{mH}$ \\
\hline Inercia & 0.0146 \\
\hline Fricción & 0 \\
\hline Pares de polos & $\mathrm{Np}=2$ \\
\hline \multirow{2}{*}{ Condición de arranque } & $\mathrm{R}_{\text {arranque }}=2 \Omega$ \\
\cline { 2 - 2 } & $\mathrm{C}_{\text {arranque }}=254.7 \mu \mathrm{F}$ \\
\hline \multirow{2}{*}{ Condición nominal } & $\mathrm{R}_{\text {estable }}=18 \Omega$ \\
\cline { 2 - 2 } & $\mathrm{C}_{\text {estable }}=21.1 \mu \mathrm{F}$ \\
\hline Velocidad síncrona & 1800 rpm en vacío \\
\hline Velocidad inicial & $95 \%$ de la síncrona \\
\hline Carga mecánica & $3 \mathrm{~N}^{*} \mathrm{~m}$ \\
\hline
\end{tabular}

De acuerdo a las características consignadas en la Tabla 1, en la Fig. $5 \mathrm{~b}$ se observa el comportamiento de la velocidad del $\mathrm{Ml}$ cuando este viene operando nominalmente y al cabo de determinado tiempo se genera una disminución de tensión de 10\% (Fig. 5a) aplicada de forma permanente.

a.

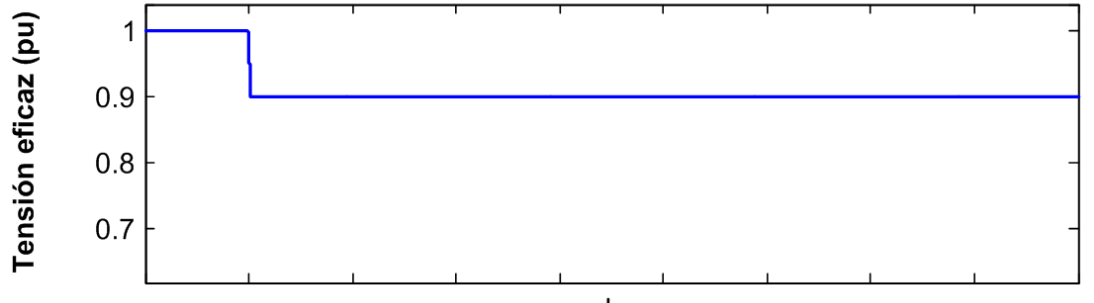

b.

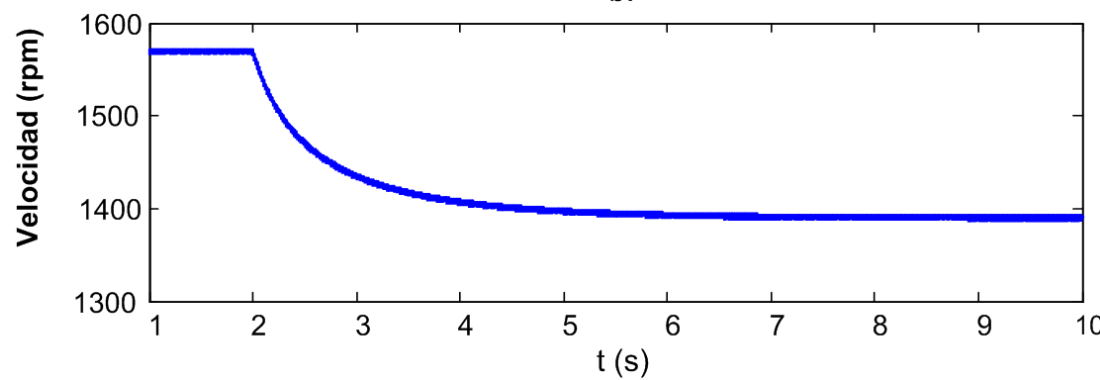

Fig. 5: Parámetros en el MI: a. tensión eficaz aplicada y b. velocidad

Con el fin de determinar el momento en el que el Ml falla durante la simulación de un hueco de tensión se implementó el sistema de la Fig. 6.

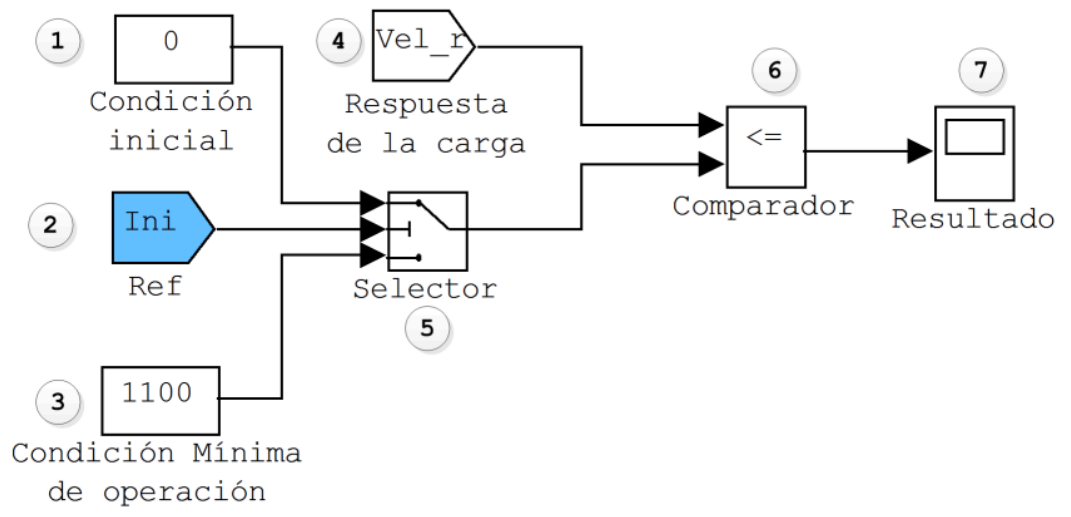

Fig. 6: Sistema para determinar el estado de la carga. 
Este sistema compara una velocidad de referencia (Condición mínima de operación) con la velocidad operación del MI (Respuesta de la carga), como resultado se obtiene una salida lógica de uno (1) cuando MI falla (velocidad del MI inferior a la velocidad de referencia), en caso contrario es cero. Las siete componentes del sistema para determinar el estado del MI (Fig. 6) se describen a continuación:

1.- Valor constante de cero que es comparado con el valor de la variable de respuesta de la carga, antes del hueco de tensión, asegurando un resultado constante igual a cero durante este intervalo de tiempo.

2.-Señal de referencia (Ref), obtenida del modelo ideal de generador (Fig. 4) durante la simulación, que establece la posición del selector en "Condición inicial", antes del hueco de tensión y en "Condición mínima de operación" cuando inicia el hueco de tensión. Esto asegura que la comparación se realice a partir de la generación del hueco de tensión.

3.- Valor de la condición mínima de operación que es comparado con el valor de respuesta de la carga (Velocidad mínima que debe tener el Ml) durante el hueco de tensión.

4.- Valor de la variable de respuesta de la carga durante la simulación (Velocidad del MI).

5.- Selector que varía su posición entre las variables de entrada ("Estado inicial" y "Condición mínima de operación") para el intervalo de tiempo establecido por la señal de referencia.

6.- Comparador del valor de la variable de respuesta de la carga con la variable de entrada indicada por el selector.

7.- Resultado de la comparación, el cual indica un valor de uno cuando el equipo falla, y cero en caso contrario.

A manera de ejemplo en este estudio se establece en forma arbitraria que la velocidad mínima del MI debe ser igual a $1100 \mathrm{rpm}$. Sin embargo, dependiendo de las características del proceso productivo al cual esté asociado el MI, se puede tomar otro valor de referencia.

En la Figura 7 se muestra el algoritmo con el que se obtiene la curva se sensibilidad del Ml, éste es una adaptación de la metodología desarrollada por Trujillo (Trujillo et al., 2007), que utiliza el modelo de ideal del generador de huecos de tensión de la Fig. 4 y el sistema de la Fig. 7, para obtener y graficar las magnitudes y duraciones de los huecos de tensión que causan fallas en el MI.

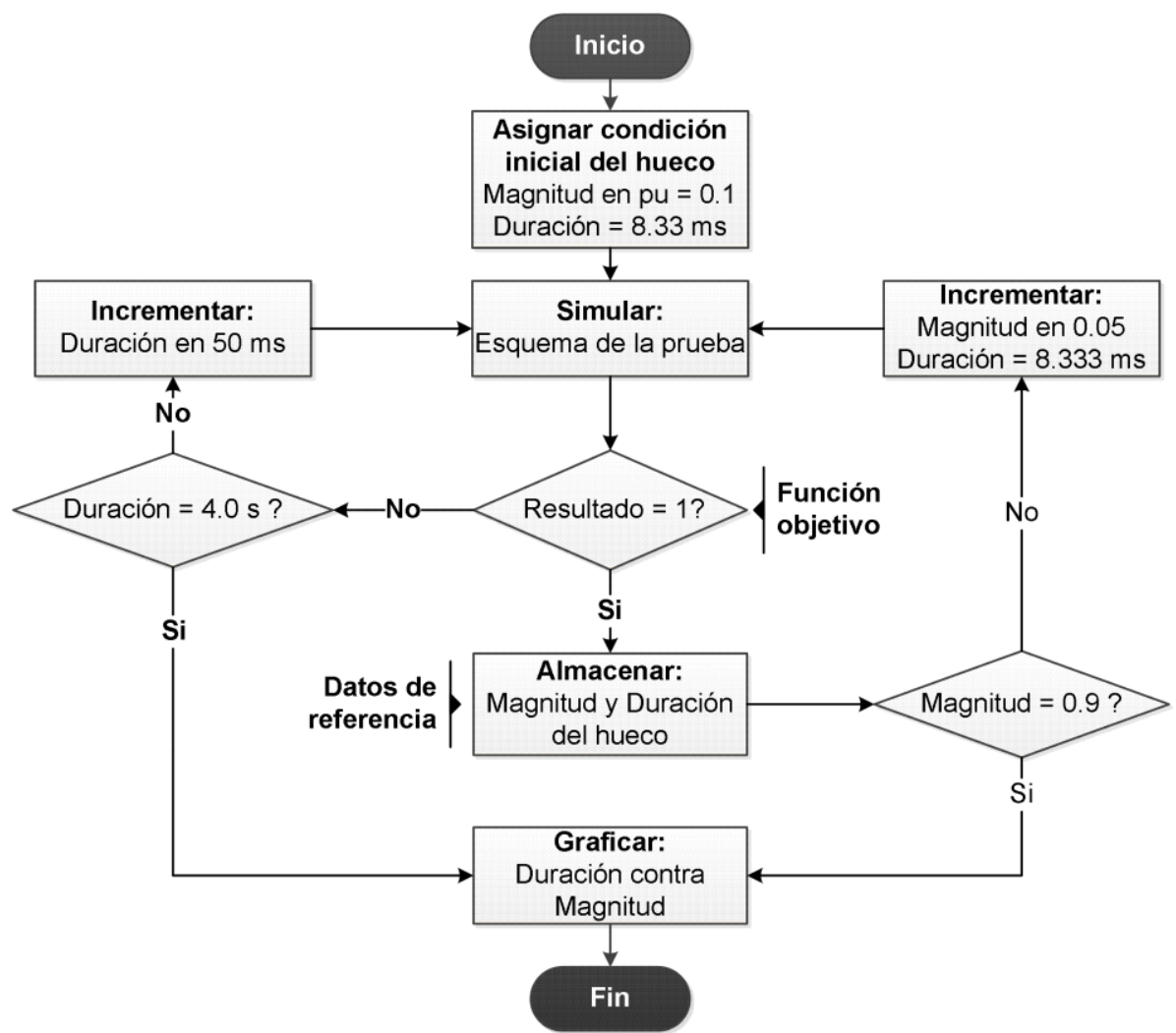

Fig. 7: Algoritmo propuesto para obtener la curva de sensibilidad del MI. 
El algoritmo ajusta la magnitud del hueco de tensión en 0.1 pu y la duración en 8.33 ms (medio ciclo), que son los límites inferiores de un hueco de tensión (IEEE Standards Coordinating Committee 22 on Power Quality, 1995). Inmediatamente, simula el esquema de la Fig. 3 y verifica la condición del MI, en caso de que no se presente falla, incrementa la duración en pasos de $50 \mathrm{~ms}$ y realiza la misma operación hasta detectar un punto de falla del MI o hasta alcanzar el tiempo límite de estado estable del MI (de acuerdo con la Fig. 5b. es de $4 \mathrm{~s}$ después de la ocurrencia del hueco de tensión). Si se encuentra un punto de falla, se almacena el valor de magnitud y duración del hueco de tensión. Posteriormente, se incrementa la magnitud del hueco de tensión en 0.05 pu y se repite el procedimiento de incremento de duración hasta detectar el punto de falla. Estos pasos se repiten hasta llegar al límite máximo de magnitud de un hueco de tensión (0.9 pu). Luego se grafican los puntos donde se presenta falla y se obtiene así la curva de sensibilidad del MI.

\section{Proceso para mejorar la precisión de la curva de sensibilidad}

El proceso de mejora de las curvas de sensibilidad se divide en dos etapas: la primera establece dos expresiones matemáticas que determinan la duración en la que el MI falla para una magnitud de un hueco de tensión especifica (Fig. 8), obtenidas a partir de un análisis de regresión estadística (Barrio et al., 2002).

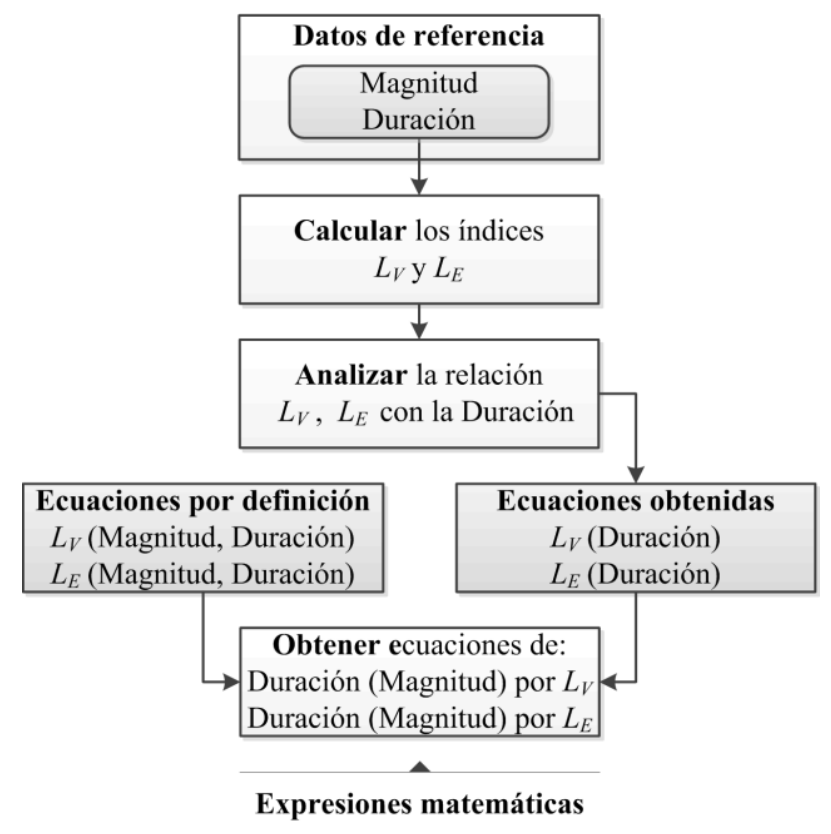

Fig. 8: Proceso para obtener el modelo matemático de la duración en función de la magnitud

En el proceso descrito en la Fig. 8, inicialmente se calculan los índices de pérdida de tensión y pérdida de energía con (3) y (4) para cada uno de los datos de referencia (valores de duración y magnitud en los que el motor falló) obtenidos con el algoritmo de la Fig. 7. A continuación se analiza la relación entre los índices $L_{V}$ y $L_{E}$ y la duración, por medio de un análisis de regresión estadística, con lo cual se obtienen dos expresiones, que describen el comportamiento de los índices mencionados con respecto a la duración de un hueco de tensión. Posteriormente las expresiones resultantes de la regresión se igualan con (3) y (4), para obtener dos ecuaciones (modelo matemático), que permiten estimar por cada magnitud de un hueco de tensión, dos duraciones en las que podría fallar el MI.

La segunda etapa corresponde al algoritmo de mejora (Fig. 9), con el cual se obtiene la curva de sensibilidad del $\mathrm{MI}$, utilizando las expresiones matemáticas establecidas en la etapa previa. El algoritmo para mejorar la curva de sensibilidad, inicia con una magnitud de hueco de tensión de 0.1 en pu. El proceso "Estimar duraciones" utiliza la expresión matemática con la cual se obtienen dos duraciones para una magnitud de un hueco de tensión.

Si las duraciones obtenidas son mayores de $4 \mathrm{~s}$ (tiempo en el que el motor ya alcanzó el estado estable de operación y no presentó falla, de acuerdo a la Fig. 5b), se ejecuta el proceso "Seleccionar: Duración crítica", de lo contrario las duraciones son almacenadas con la magnitud correspondiente y se denominan datos estimados. La constante $K$ en el algoritmo (Fig. 9) representa la mayor de las magnitudes de los huecos de tensión almacenados en los datos de referencia (Obtenidos al aplicar el algoritmo de la Fig. 7). La constante $X$ representa el incremento de la magnitud en pu. Cuando la magnitud del hueco de tensión es mayor o igual $K$, se lleva a cabo el proceso "Seleccionar: Duración crítica", en caso contrario, la magnitud del hueco de tensión se incrementa en $X$ en pu, y vuelve al proceso "Estimar: Duraciones". 
El proceso "Seleccionar: Duración crítica" consiste en elegir la menor duración para cada magnitud de un hueco de tensión, entre los datos estimados y los datos de referencia. Finalmente se realiza la gráfica de duración versus magnitud, y se obtiene la curva de sensibilidad con incrementos en magnitud de $X$ en pu.

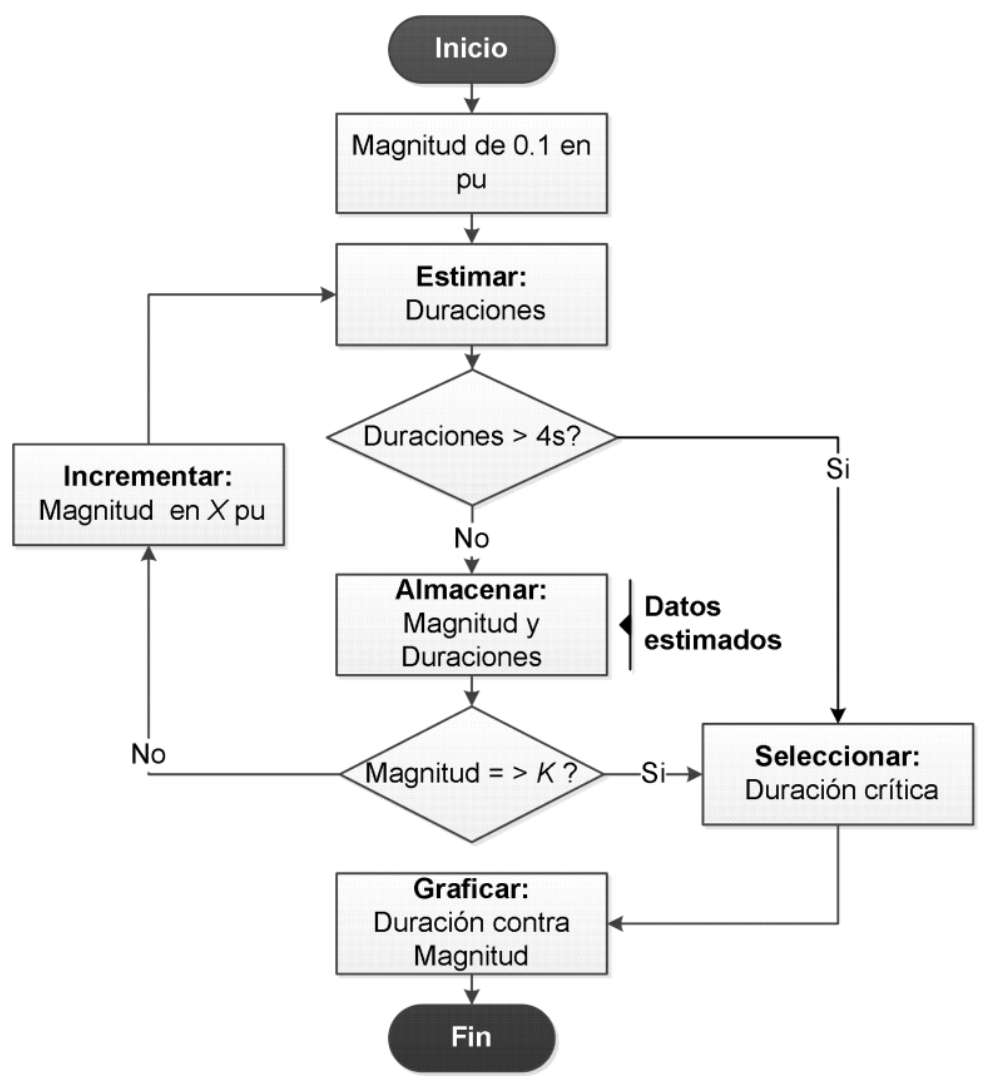

Fig. 9: Algoritmo implementado para mejorar la curva de sensibilidad

\section{RESULTADOS DE LA EVALUACIÓN DE LA INMUNIDAD DEL MI}

La Fig. 10 muestra el resultado de la curva de sensibilidad del Ml, obtenida con el algoritmo de la Fig. 7, los puntos rojos de la curva representan los datos de referencia (puntos especifico donde se detectó falla del $\mathrm{MI})$.

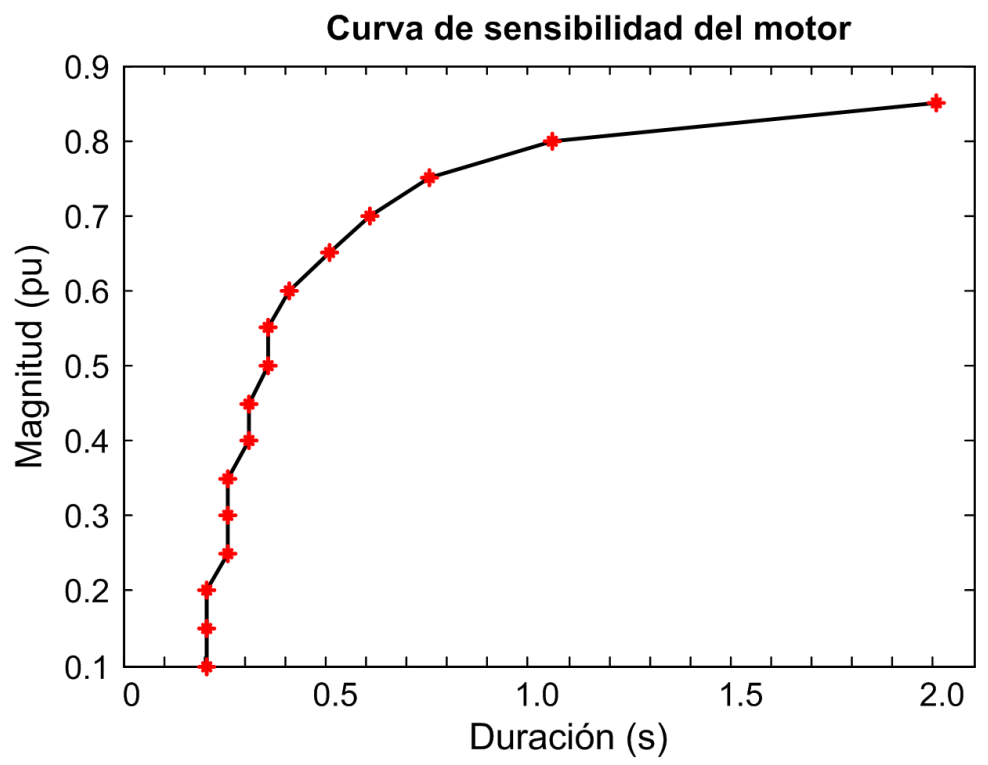

Fig. 10: Curva de sensibilidad del MI 
Los resultados de la Fig. 10, se interpretan de la siguiente forma: El motor de inducción con las condiciones establecidas en la sección de Metodología, falla para las magnitudes y duración de huecos de tensión que se encuentren debajo de la curva mostrada, así por ejemplo, un hueco de tensión con magnitud de 0.6 en pu y una duración de $1.0 \mathrm{~s}$, provocaría falla en el Ml durante el proceso (para el caso en evaluación, velocidad del MI menor a $1100 \mathrm{rpm}$ ). Para las magnitudes y duraciones que se encuentre por encima de la curva, el MI no presentará falla, por lo tanto el hueco de tensión con magnitud de 0.6 en pu, con una duración de $0.2 \mathrm{~s}$, no produciría falla en el MI.

Aplicando el proceso de la Fig. 8, para obtener las expresiones matemáticas, se calculan los valores de referencia de los índices de estimación que se presentan en la Tabla 2.

Tabla 2: Resultados de los índices de pérdida de tensión y pérdida de energía

\begin{tabular}{|c|c|c|c|}
\hline Magnitud (pu) & Duración (s) & $L_{V}$ & $L_{E}$ \\
\hline 0.100 & 0.208 & 0.187 & 0.206 \\
\hline 0.150 & 0.208 & 0.177 & 0.204 \\
\hline 0.200 & 0.208 & 0.167 & 0.200 \\
\hline 0.250 & 0.258 & 0.194 & 0.242 \\
\hline 0.300 & 0.258 & 0.181 & 0.235 \\
\hline 0.350 & 0.258 & 0.168 & 0.227 \\
\hline 0.400 & 0.308 & 0.185 & 0.259 \\
\hline 0.450 & 0.308 & 0.170 & 0.246 \\
\hline 0.500 & 0.358 & 0.179 & 0.269 \\
\hline 0.550 & 0.358 & 0.161 & 0.250 \\
\hline 0.600 & 0.408 & 0.163 & 0.261 \\
\hline 0.650 & 0.508 & 0.178 & 0.294 \\
\hline 0.700 & 0.608 & 0.182 & 0.310 \\
\hline 0.750 & 0.758 & 0.190 & 0.332 \\
\hline 0.800 & 1.058 & 0.212 & 0.381 \\
\hline 0.850 & 2.008 & 0.301 & 0.557 \\
\hline
\end{tabular}

La relación entre los índices $L_{V}$ y $L_{E}$ con respecto a la duración de los huecos, se observa en la Fig. 11, que muestra en ambos casos una tendencia lineal. Aplicando el análisis de regresión para la tendencia lineal entre los índices de estimación y la duración, se obtienen los resultados de la Tabla 3.

a. Índice de pérdida de tensión
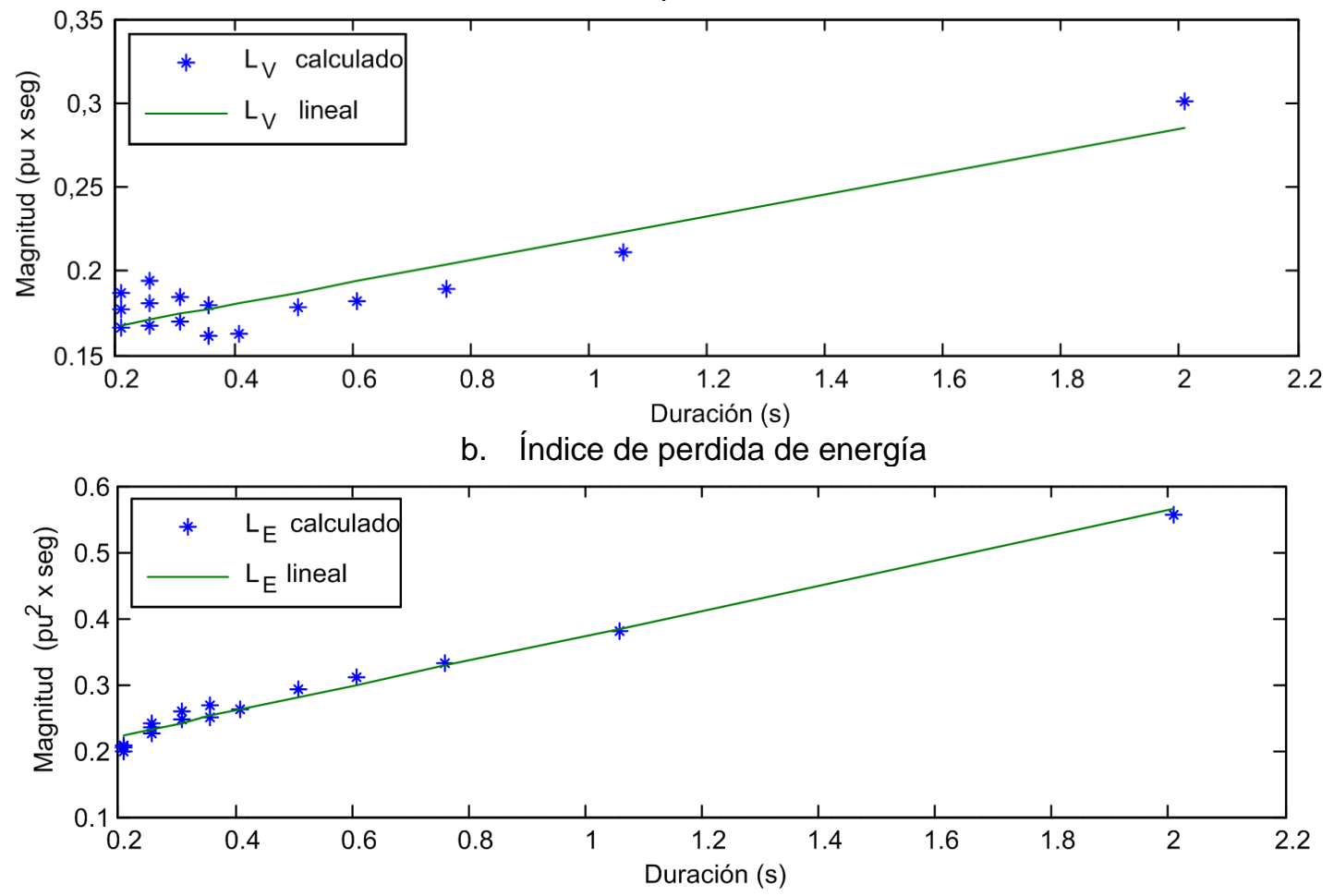

Fig. 11: Relación entre la duración y los índices de estimación: a. pérdida de tensión y b. pérdida de energía 
El coeficiente de correlación múltiple y el coeficiente de determinación expresados en la Tabla 3, establecen que el grado de asociación lineal entre los valores de los índices de estimación y la duración es alto (de $91.6 \%$ y $99 \%$ para $L_{V}$ y $L_{E}$ respectivamente), y que además, las ecuaciones lineales que se obtiene a partir de esos datos, son confiables, por lo tanto el modelo lineal es una buena representación de los índices $L_{V}$ y $L_{E}$ versus la duración.

Tabla 3: Resultado del análisis de regresión lineal

\begin{tabular}{|l|c|c|}
\hline \multicolumn{1}{|c|}{ Índice } & $L_{V}$ & $L_{E}$ \\
\hline \multicolumn{2}{|l|}{ Estadísticas de la regresión } \\
\hline Coeficiente de correlación múltiple & 0.916 & 0.990 \\
\hline Coeficiente de determinación $\mathrm{R}^{2}$ & 0.840 & 0.980 \\
\hline $\mathrm{R}^{2}$ ajustado & 0.828 & 0.979 \\
\hline Error típico & 0.014 & 0.013 \\
\hline Coeficientes & \\
\hline Intercepción & 0.154 & 0.184 \\
\hline Pendiente & 0.065 & 0.189 \\
\hline
\end{tabular}

Como resultado de la regresión se obtienen las ecuaciones los índices $L_{V}$ y $L_{E}$ en función de la duración.

$\mathrm{L}_{V}=0.065^{\star} \Delta t+0.154$

$L_{E}=0.189^{*} \Delta t+0.184$

Igualando (5) y (6) con (3) y (4), se obtienen las ecuaciones para determinar las duraciones en las que probablemente un hueco de tensión de cierta magnitud podría causar falla en el MI.

$\Delta \mathrm{t}=\frac{0.154}{1-\mathrm{V}_{\mathrm{pu}}-0.065}$

$\Delta t=\frac{0.199}{1-V_{p u}{ }^{2}-0.180}$

Si al aplicar (7) y (8), con una magnitud de hueco de tensión, se obtienen duraciones mayores a $4 \mathrm{~s}$ (tiempo en el que el $\mathrm{Ml}$ alcanza el estado estable), significa que el MI no falla frente a un hueco de tensión de dicha magnitud. De otro lado, aplicando el algoritmo para mejorar la curva de sensibilidad (Fig. 9) en un rango de magnitud de huecos de tensión de 0.1 a 0.85 pu (Rango de referencia), con incrementos en magnitud $(X)$ de 0.001 en pu, se obtiene como resultado la Fig. 12.

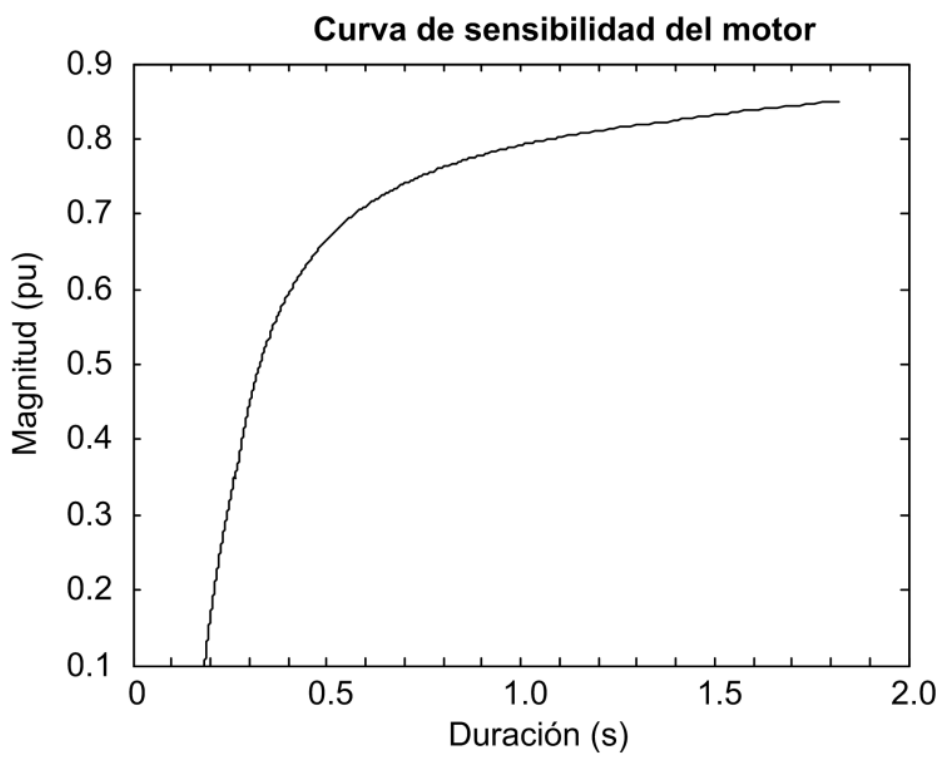

Fig. 12: Curva de sensibilidad del MI mejorada 
Finalmente, para validar la nueva curva de sensibilidad, se seleccionan aleatoriamente 20 huecos de tensión dentro de los valores propios de la curva, y se simula cada uno de estos valores con el esquema de la Fig. 3. Los resultados se tabulan en la Tabla 4. De los 20 huecos de tensión seleccionados, 5 de ellos no causaron falla en el $\mathrm{Ml}$, en dichos puntos se evalúa la diferencia de tiempo con respecto a la duración del hueco que causa falla. Esto se logra incrementando la duración estimada en pasos de $1 \mathrm{~ms}$ hasta detectar la falla, el resultado se observa en la columna 4 de la Tabla 4, en dichos casos el error es inferior al $3 \%$ (Columna 5).

Tabla 4: Resultado de la evaluación de la predicción de la curva de sensibilidad

\begin{tabular}{|c|c|c|c|c|}
\hline $\begin{array}{c}\text { Magnitud } \\
(\mathrm{pu})\end{array}$ & $\begin{array}{c}\text { Duración } \\
\text { Estimada }(\mathrm{s})\end{array}$ & $\begin{array}{c}\text { Resultado de la } \\
\text { prueba }\end{array}$ & $\begin{array}{c}\text { Duración en la que } \\
\text { Ml falla }(\mathrm{s})\end{array}$ & $\begin{array}{c}\text { Error de } \\
\text { estimación }\end{array}$ \\
\hline 0.107 & 0.186 & No falla & 0.19 & $2.11 \%$ \\
\hline 0.192 & 0.208 & Falla & & $1.40 \%$ \\
\hline 0.204 & 0.211 & No falla & 0.214 & \\
\hline 0.225 & 0.217 & Falla & & \\
\hline 0.263 & 0.230 & Falla & & \\
\hline 0.315 & 0.249 & Falla & & \\
\hline 0.357 & 0.267 & Falla & & \\
\hline 0.389 & 0.279 & Falla & & \\
\hline 0.411 & 0.287 & Falla & & \\
\hline 0.433 & 0.295 & Falla & & \\
\hline 0.526 & 0.344 & Falla & & $0.12 \%$ \\
\hline 0.539 & 0.353 & Falla & & $0.14 \%$ \\
\hline 0.597 & 0.405 & No falla & & \\
\hline 0.626 & 0.439 & Falla & & \\
\hline 0.644 & 0.464 & No falla & 0.406 & \\
\hline 0.664 & 0.497 & No falla & 0.498 & \\
\hline 0.760 & 0.789 & falla & & \\
\hline 0.774 & 0.869 & Falla & & \\
\hline 0.798 & 1.058 & Falla & & \\
\hline 0.822 & 1.363 & Falla & & \\
\hline
\end{tabular}

\section{CONCLUSIONES}

Los principales aportes del trabajo de investigación realizado y que se plasma en este artículo radica en el diseñó un modelo de generador monofásico de huecos de tensión, en la implementación de algoritmos con el que se obtiene, modela y mejora la curva de sensibilidad del MI con respecto a las caídas de tensión, así como la implementación de una metodología, con el que se podrían implementar protocolos de evaluación de inmunidad de motores de inducción a nivel industrial frente a huecos de tensión.

La metodología propone una guía práctica para la obtención de la curva de sensibilidad de un equipo eléctrico-electrónico, para ello se utilizan índices de pérdida de tensión y pérdida de energía, los cuales facilitan la obtención de una curva sensibilidad estimada, con un margen de error bajo, a partir de pocos puntos de referencia. La curva de sensibilidad es una herramienta adecuada para la evaluación del rendimiento de los equipos frente a los huecos de tensión, dado que éstos son afectados principalmente por la magnitud y la duración.

De las consideraciones importantes a tener en cuenta es que el número de huecos de tensión al cual se debe someter un MI real para obtener su curva de sensibilidad frente a huecos de tensión, disminuye cuando se aplica la metodología expuesta, al igual que el tiempo de realización de la prueba, ya que se requieren pocos huecos de tensión para estimar una curva de sensibilidad; sin embargo, para obtener referencias de comportamiento en todo el rango de magnitudes de los huecos de tensión, los puntos de referencia deben obtenerse utilizando magnitudes distribuidas entre el 0.1 y el 0.9 en pu.

\section{REFERENCIAS}

Afonso J.; Batista J.; Sepúlveda M y Martins J., Sistema Digital de Bajo Coste para la Monitorización de la Calidad de Energía Eléctrica, Información Tecnológica, 18 (4), 15-23 (2007).

Bollen, M.; Sabin, D. D. y Thallam, R. S. Voltage-sag indices - recent developments in IEEE PI564 task force. Quality and Security of Electric Power Delivery Systems, CIGRE/IEEE PES International Symposium. 34 - 41, 8-10 de Octubre, (2003). 
Bollen, M. 2000. Understanding Power Quality Problems: Voltage Sags and Interruptions, 1ra ed., IEEE Press on Power Engineering, (2000).

Caicedo, J.; Navarro, F.; Rivas, E. y Santamaría, F. Voltage Sag Characterization with Matlab/Simulink, 2012 IEEE Workshop on Engineering applications, 1-6, Bogotá, Colombia, 2 a 4 de Mayo (2012).

Caicedo, J.; Navarro, F.; Rivas, E. y Santamaría, F. State of the Art and New Developments in Voltage Sag Immunity, Ingeniería e Investigación, 31(2), 81-87 (2011).

CIGRE/CIRED/UIE Joint Working Group C4.110. Voltage Dip Immunity of Equipment and Installations. (2010).

Conrad, L. y Bollen, M. Voltage sag coordination for reliable plant operation. IEEE Transactions on Industry Applications, 33(6), 1459-1464 (1997).

IEEE Std, 1159, IEEE Recommended Practice for Monitoring Electric Power Quality. pag. 12, 16, (1995).

Gomez J.; Morcos M.; Reineri C. y Campetelli G., Behavior of Induction Motor Due to Voltage Sags and Short Interruptions, IEEE Transactions on Power Delivery 17(2), 799-808 (2002).

Key, T., Diagnosing Power Quality-Related Computer Problems. IEEE Transactions on Industry Applications, IA-15 (4), 381-393 (1979).

Leborgne, R.; Filho, J.M.C.; de Abreu, J.P.G.; Oliveira, T.C. ; Postal, A.A. y Zaparoli, L.H., Alternative Methodology for Characterization of industrial process sensitivity to voltage sags. 2003 IEEE Bologna Power Tech Conference Proceedings, (2003).

Macri, M.G. y Benedetti, M., Análisis multirresolución del motor trifásico de inducción sometido a huecos de tensión, Revista Chilena de Ingeniería, 20(1), 66-79 (2012).

McGranaghan, M.; Mueller, D.R. y Samotyj, M.J., Voltage sags in industrial systems, IEEE Transactions on Industry Applications, 29(2), 397-403 (1993).

Senturk O. y Hava A., A Simple Sag Generator Using SSRs. IEEE Transactions on Industry Applications 48(1), 172-180 (2012).

Su C.; Chen C. y Lee C., Fast Evaluation Methods for Voltage Sags in Ship Electrical Power Systems, IEEE Transactions on Industry Applications 49(1), 233- 241 (2013).

Suriñach J.; Clar M. y Del Barrio T., Modelo de Regresion Lineal Multiple: especificación, estimación y contraste, Barcelona: UOC Papers,(2002).

Tenaga Nasional Berhad. Voltage Sag Solutions for Industrial Customers. Power Quality Guidebook. (2007).

Trujillo, C.; Guacaneme, J.; Torres H. y Pavas A., Methodology for Assessing the Impact of Short-Term Voltage Variations (Sags) on Power Electronic Equipment. 9th International Conference. Electrical Power Quality and Utilisation., Barcelona, España, 9-11 de octubre, (2007)

Wang X.; Yong J.; Xu W. y Freitas W., Practical Power Quality Charts for Motor Starting Assessment IEEE Transactions on Power Delivery 26(2), 799-808 (2011).

Wang Z.; Xiaoyu W. y Chung C., An Analytical Method for Calculating Critical Voltage Sag Clearance Time of Induction Motors IEEE Transactions on Power Delivery 27(4), 2412-2414 (2012). 